\title{
RESEARCH
}

Open Access

\section{TFEB-NF-kB inflammatory signaling axis: a novel therapeutic pathway of Dihydrotanshinone I in doxorubicin- induced cardiotoxicity}

Xiaoping Wang ${ }^{1 \dagger}$, Qiyan Wang ${ }^{1+}$, Weili Li ${ }^{1}$, Qian Zhang ${ }^{2}$, Yanyan Jiang ${ }^{1}$, Dongqing Guo ${ }^{1}$, Xiaoqian Sun ${ }^{2}$, Wenji Lu², Chun $\mathrm{Li}^{3^{*}}$ and Yong Wang ${ }^{1,2^{*}}$

\begin{abstract}
Background: Doxorubicin is effective in a variety of solid and hematological malignancies. Unfortunately, clinical application of doxorubicin is limited due to a cumulative dose-dependent cardiotoxicity. Dihydrotanshinone I (DHT) is a natural product from Salvia miltiorrhiza Bunge with multiple anti-tumor activity and anti-inflammation effects. However, its anti-doxorubicin-induced cardiotoxicity (DIC) effect, either in vivo or in vitro, has not been elucidated yet. This study aims to explore the anti-inflammation effects of DHT against DIC, and to elucidate the potential regulatory mechanism.

Methods: Effects of DHT on DIC were assessed in zebrafish, C57BL/6 mice and H9C2 cardiomyocytes. Echocardiography, histological examination, flow cytometry, immunochemistry and immunofluorescence were utilized to evaluate cardio-protective effects and anti-inflammation effects. mTOR agonist and lentivirus vector carrying GFP-TFEB were applied to explore the regulatory signaling pathway.
\end{abstract}

Results: DHT improved cardiac function via inhibiting the activation of M1 macrophages and the excessive release of pro-inflammatory cytokines both in vivo and in vitro. The activation and nuclear localization of NF-kB were suppressed by DHT, and the effect was abolished by mTOR agonist with concomitant reduced expression of nuclear TFEB. Furthermore, reduced expression of nuclear TFEB is accompanied by up-regulated phosphorylation of $I \mathrm{KK}$ / $\beta$ and NF-KB, while TFEB overexpression reversed these changes. Intriguingly, DHT could upregulate nuclear expression of TFEB and reduce expressions of $\mathrm{p}-\mathrm{KKKa} / \mathrm{\beta}$ and $\mathrm{p}-\mathrm{NF}-\mathrm{KB}$.

Conclusions: Our results demonstrated that DHT can be applied as a novel cardioprotective compound in the antiinflammation management of DIC via mTOR-TFEB-NF-KB signaling pathway. The current study implicates TFEB-IKKNF-KB signaling axis as a previously undescribed, druggable pathway for DIC.

Keywords: TFEB-NF-KB, Inflammation, Doxorubicin, Cardiotoxicity, Dihydrotanshinone I

\footnotetext{
*Correspondence: lichun19850204@163.com; doctor_wangyong@163.com

+Xiaoping Wang and Qiyan Wang contributed equally to this work.

${ }^{3}$ School of Chinese Materia Medica, Beijing University of Chinese Medicine, Beijing 100029, China

${ }^{1}$ School of Life Science, Beijing University of Chinese Medicine, Beijing 100029, China

Full list of author information is available at the end of the article
}

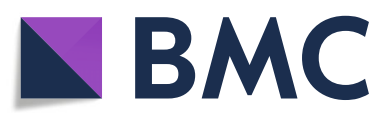

( The Author(s). 2020 Open Access This article is licensed under a Creative Commons Attribution 4.0 International License, which permits use, sharing, adaptation, distribution and reproduction in any medium or format, as long as you give appropriate credit to the original author(s) and the source, provide a link to the Creative Commons licence, and indicate if changes were made. The images or other third party material in this article are included in the article's Creative Commons licence, unless indicated otherwise in a credit line to the material. If material is not included in the article's Creative Commons licence and your intended use is not permitted by statutory regulation or exceeds the permitted use, you will need to obtain permission directly from the copyright holder. To view a copy of this licence, visit http://creativecommons.org/licenses/by/4.0/. The Creative Commons Public Domain Dedication waiver (http://creativecommons.org/publicdomain/zero/1.0/) applies to the data made available in this article, unless otherwise stated in a credit line to the data. 


\section{Background}

Doxorubicin (DOX) is one of the most commonly used chemotherapeutic drugs for a wide range of cancers, such as leukemia, soft tissue sarcomas and breast cancer [1]. However, the rate of DOX-induced cardiotoxicity (DIC) is reported to be as high as $57 \%$, and the mortality rate from these heart diseases is reported to be 8.2 times higher than that in healthy people [2]. Currently, dexrazoxane is the only FDA approved drug for the protection against cardiotoxicity. However, the Committee for Medicinal Products for Human Use (CHMP) in the UK has published the outcome of a referral that recommends several restrictions on dexrazoxane use in both children and adults with cancer. Therefore, there is an urgent need to identify the underlying mechanism of DIC and novel therapeutic agents that can prevent and/or reverse DOX-induced cardiovascular adverse effects. Recently, inflammation induced by doxorubicin has been identified as a high risk factor for developing heart failure and drugs with anti-inflammatory properties are attractive therapeutics for alleviating DIC $[3,4]$.

Nuclear factor- $\kappa B$ (NF- $k B$ )-mediated inflammatory pathway is a classic signaling cascade that controls the expressions of pro-inflammatory genes in multiple types of cells. In the resting state, inactive NF$\kappa B$ is sequestered in the cytoplasm by ІкB (inhibitor of NF- $\mathrm{kB}$ ). Under stimulation of pro-inflammatory signals, IKKs (ІКB kinases) are activated which phosphorylates $І$ І $B$ on serine residues. Phosphorylated $\mathrm{I} \kappa \mathrm{B}$ will be degraded and active NF- $\mathrm{KB}$ dimers will be released and translocated into nucleus, inducing expressions of inflammatory cytokines, such as tumor necrosis factor- $\alpha$ (TNF- $\alpha$ ) and interleukin 8 (IL-8). Several studies have confirmed that levels of inflammatory cytokines are increased in myocardial cells under doxorubicin-stimulated condition $[5,6]$. Furthermore, hyper-activation of NF- $\kappa B$ is implicated in inflammatory responses in DIC $[5,7]$. Nevertheless, the regulatory mechanisms of NF- $\mathrm{KB}$ transcriptional activity in DIC are still unclear.

The mammalian target of rapamycin (mTOR) is an atypical serine/threonine kinase, which regulates physiological homeostasis at the cellular and holistic level. Transcription factor EB (TFEB) plays a master role in governing basic cellular processes [8]. The mTOR-mediated phosphorylation negatively regulates TFEB nuclear translocation and activity [9]. It is of note that rapamycin analogues are therapeutically used as immuno-suppressants, and TFEB was also validated to have potential anti-inflammatory effect [10]. In this study, we further investigated whether mTOR-TFEB pathway plays a role in DIC-related inflammation. A profound understanding of this mechanism will provide a basis for discovery of novel targets as well as the therapeutic agents in alleviating DIC.

Salvia miltiorrhiza Bunge has been widely applied in traditional Chinese medicine (TCM) for a long history in the treatment of various inflammatory diseases [11]. As a dietary supplement, it is the first TCM to be documented in USP 37-NF32. Dihydrotanshinone I (DHT) is the one of major active diterpenes in the liposoluble extract [12]. Studies have shown that DHT possesses a variety of anti-tumor and anti-inflammatory properties [13-16]. However, whether DHT could alleviate DIC and whether the protective effect is medicated by the anti-inflammatory pathway remain elusive. In this study, we established an in vitro DOX-stimulated $\mathrm{H} 9 \mathrm{C} 2$ model and an in vivo DIC mice model. The anti-inflammatory mechanism of DHT was investigated. This study will provide insight into the anti-inflammatory strategies and drug combination therapy in the management of DIC.

\section{Methods \\ Reagents and chemicals}

Saline was purchased from SiYao Co., Ltd. (Shijiazhuang, China). Doxorubicin was bought from ApexBio Technology LLC (Houston, TX, United States). DHT was purchased from Shanghai Shidande SDHTdard Technical Service Co., Ltd. (Shanghai, China). Rapamycin was purchased from Sangong Pharmaceutical Co., Ltd. (Shanghai, China). 4\% paraformaldehyde was from Beijing Applygen Technology Inc. (Beijing, China). Dimethyl sulfoxide (DMSO) was purchased from Sigma-Aldrich LLC (Shanghai, China). PDTC was purchased from Abmole China Branch. MHY1485 was purchased from Selleck (Houston, TX, United States). Dulbecco's Modification of Eagle's Medium (DMEM), Fetal Bovine Serum (FBS), Penicillin, streptomycin, $0.1 \mathrm{~mol} / \mathrm{L}$ sodium cacodylate buffer, sodium carboxymethylcellulose and DAPI were purchased from Beijing BioDee Biotechnology Co., Ltd. (Beijing, China). GFP-TFEB lentiviral vector were bought from Hanbio Technology Co., Ltd. (Shanghai, China). All other chemicals were purchased from commercial sources.

\section{Establishment of DIC model in Zebrafish, in mice and pharmacological treatments}

The DIC model in zebrafish was established according to the methods described in a previous study [17]. Briefly, after the heart had formed and circulation had begun 1 day postfertilization (dpf), zebrafish were treated with $100 \mu \mathrm{M}$ DOX or/and $10 \mathrm{nM}$ DHT, and phenotypic changes, including fraction shortening (FS), the blood speed of the tail vein, heart rate and survival rate were respectively assessed at three $\mathrm{dpf}$.

C57BL/6 male mice $(18 \mathrm{~g} \pm 2 \mathrm{~g})$ were purchased from Beijing SPF Biotechnology Co., Ltd., China (Beijing, 
China). All mice were housed at temperature $22 \pm 2{ }^{\circ} \mathrm{C}$, with proper humidity, lighting $(12 \mathrm{~h}$ light $/ 12 \mathrm{~h}$ dark cycle), and free access to food and water. All mice were randomized into four groups as follows: 16 mice in the DOX group, DHT-treated group or positive drug (Rapamycin, Rapa)-treated group, respectively; 10 mice in Saline group. DIC model was induced via tail vein injection with DOX ( $5 \mathrm{mg} \cdot \mathrm{kg}^{-1}$ ) once weekly for 4 weeks and sham group was given with saline $(0.9 \% \mathrm{NaCl})$ at the same time. This mice model has been described in previous study [18]. Besides, as the positive control drug, Rapamycin, an inhibitor of mTOR, was widely reported to be able to alleviate cardiac inflammation $[19,20]$. Each drug was orally and daily administered for 4 weeks starting on 1 week after the last DOX injection. The doses of DHT $\left(20 \mathrm{mg} \cdot \mathrm{kg}^{-1}\right)$ and Rapamycin (2.81 $\mathrm{mg} \cdot \mathrm{kg}^{-1}$ ) were determined based on previous studies $[21,22]$. Given that DHT and Rapamycin are both poorly soluble in water, we applied $0.5 \%$ aqueous solution of a sodium carboxymethylcellulose (CMC) as the suitable vehicle as previous literature described. And the Saline group and the DOX group received the same volume of vehicle. All animal procedures were carried out in accordance with the Guide for the Care and Use of Laboratory Animals (NIH Publications No.85-23) and with Beijing University of Chinese Medicine Animal Care Committee (approve code BUCM-4-2,018,101,5044068). After study, all mice were anaesthetized by isoflurane inhalation $2 \%$ and then euthanized by cervical dislocation.

\section{Echocardiographic assessment of cardiac functions}

After 4 weeks' administration, cardiac function was examined by Transthoracic echocardiography (Vevo TM 2100; Visual Sonics, Canada). Left ventricular enddiastolic volume (LVEDV), left ventricular end-systolic volume (LVESV), left ventricular end-diastolic dimension (LVEDD) and left ventricular end-systolic dimension (LVESD) were assessed for at least three uninterrupted cardiac cycles. Then mice were sacrificed, heart and blood were collected.

\section{Histological examination}

For histological analysis, hearts were immersed in 4\% paraformaldehyde for at least $24 \mathrm{~h}$, then were embedded in paraffin and cut into $4 \mu \mathrm{m}$ serial slices. Paraffin sections were stained with Hematoxylin-Eosin (HE) and were monitored under an optical microscope at $400 \times$ magnification.

\section{Malondialdehyde (MDA) assay and superoxide dismutase (SOD) assay}

MDA and SOD content commonly reflect the level of oxidative stress. Evidences showed that oxidative stress and inflammation are regarded as essential partners presenting simultaneously and interact with each other in diverse pathological conditions. MDA content and SOD activity in plasma were detected by following the instructions of commercially available kits (Jiancheng, Nanjing, China). MDA level was expressed as $\mathrm{nmol} / \mathrm{L}$ in plasma. SOD activity was expressed as $\mathrm{U} / \mathrm{mL}$ in plasma.

\section{Immunochemistry}

The slices were dewaxed with xylene and gradient alcohol hydration, then were added with drops of $3 \% \mathrm{H}_{2} \mathrm{O}_{2}$ for resting $15 \mathrm{~min}$ to block endogenous peroxidase. After washing with phosphate buffered saline (PBS) for three times, the cartilage slices were added with heated sodium citrate buffer for microwaving 6 min to antigen repair, which was repeated twice. After cooling and washing with PBS, the cartilage slices were treated with $5 \%$ goat serum for $10 \mathrm{~min}$, to obstruct the binding of nonspecific antibody, then added with drops of the first antibodies of TNF- $\alpha$. Later the slices were incubated at $37^{\circ} \mathrm{C}$ for about $30 \mathrm{~min}$ and washed with PBS. After reacting with the second antibody, the slices were incubated at $37^{\circ} \mathrm{C}$ for another $30 \mathrm{~min}$ and washed with PBS, later followed by staining for $10 \mathrm{~min}$ with diaminobenzidine $(D A B)$, washing with running pure water, restaining with hematoxylin and mounting with neutral balsam. Finally, we observed the images under a microscope and picture-taking.

\section{Flow cytometry}

Flow cytometry was performed referring to literature concerned [23]. Hearts from mice (three mice per group) were minced into small pieces around $1 \mathrm{~mm}^{3}$ followed by digestion with 40\% collagenase II (17101-015; Invitrogen, CA, United States) and 0.25\% Trypsin (17104-019; Invitrogen, CA, United States) for $10 \mathrm{~min}$. To disperse solid tissue into single cells, the enzymes (mainly collagenase and protease) were used to digest collagen fibers and elastic fibers, and to hydrolyze proteins and mucopolysaccharide substances that tight junction structure of tissue cells $[24,25]$. Then cells were put into centrifuge at a force of $300 \mathrm{~g}$ for $5 \mathrm{~min}$. After washing two times with PBS, cells were resuspended with $200 \mu \mathrm{L}$ PBS, then stained with $0.75 \mu \mathrm{g}$ anti-CD11b, $1 \mu \mathrm{g}$ anti-F4/80, $1 \mu \mathrm{g}$ anti-CD86 and $1 \mu \mathrm{g}$ anti-CD206 antibodies. Followed by examining on a FACS Canto II flow cytometer (BD Biosciences). Data were analyzed by using the FlowJo software (FlowJo, LLC, Ashland, OR, USA). The antibodies were listed in Table 1.

\section{Establishment of LPS-induced RAW264.7 cell model and pharmacological treatments}

RAW 264.7 macrophages in our study were obtained from China Infrastructure of Cell Line Resources. RAW 264.7 macrophages were cultured in DMEM supplemented with 
Table 1 Antibodies used in Flow cytometry

\begin{tabular}{ll}
\hline Protein & Antibody \\
\hline PE-Cy7 Rat Anti-CD 11b Clone M1/70 & 561,$098 ;$ BD PMG, United States \\
F4/80 (BM8.1) Rat mAb (APC Conjugate) & 86,$007 ;$ Cell Signaling Technology, Germany \\
PE anti-mouse CD86 & 105,$105 ;$ BioLegend, United States \\
FITC anti-mouse CD206 (MMR) Antibody & 141,$703 ;$ BioLegend, United States \\
\hline
\end{tabular}

$10 \% \mathrm{FBS}$ at $37^{\circ} \mathrm{C}$ in a humidified atmosphere $\left(5 \% \mathrm{CO}_{2}\right.$ and $9 \% \mathrm{O}_{2}$ ). To evaluate the effects of DHT on LPSinduced RAW264.7, cells were stimulated with LPS (1 $\mu \mathrm{g}$ / $\mathrm{mL}$ ) for $24 \mathrm{~h}$ with or without DHT (added to media $2 \mathrm{~h}$ before treating with LPS). The concentrations of DHT in culture media in different cell groups were 10, 50 and 100 $\mathrm{nM}$, respectively.

\section{Detection for TNF-a in the supernatant}

Cell supernatants were collected from LPS-induced RAW 264.7 (with or without DHT pretreatment). The concentrations of TNF- $\alpha$ and IL- $1 \beta$ in the supernatants were determined by using ELISA kits bought from Boster Biological Technology co.ltd and the manufacturer's instructions were followed. TNF- $\alpha$ and IL-1 $\beta$ levels were expressed as $\mathrm{pg} / \mathrm{mL}$.

\section{Establishment of DOX-stimulated $\mathrm{H} 9 \mathrm{C} 2$ cell model and pharmacological treatments}

H9C2 cells in our study were obtained from China Infrastructure of Cell Line Resources. The establishment of DOX-stimulated $\mathrm{H} 9 \mathrm{C} 2$ cell model has been mentioned in our previous study [26]. For pharmacological treatments, we set up various groups: Control group, DOX group (with DOX $1 \mu \mathrm{M}$ ), DOX + DHT group (with DHT $10 \mathrm{nM}$ ), DOX + DHT + MHY1485 group (with DHT 10 $\mathrm{nM}$ and MHY1485 $5 \mu \mathrm{M}$ ) and DOX + PDTC group (with PDTC $100 \mathrm{nM}$ ). For western blot analysis, H9C2 cells were cultured in $10 \mathrm{~cm}$ petri dishes. MHY1485, an agonist of mTOR, was applied to verified whether DHT can inhibit mTOR, sequentially lead to downregulation of NF- $\mathrm{kB}$-mediated inflammatory response. PDTC, a NF$\kappa \mathrm{B}$ inhibitor, was set as the positive drug in vitro experiment.

\section{GFP-TFEB lentiviral vector transfection}

The specific methods have been described in our previous study [26].

Assessment of apoptosis by a Hoechst 33258 staining kit H9C2 cells were fixed with ice-cold $4 \%$ paraformaldehyde for $20 \mathrm{~min}$ after washed with $0.1 \mathrm{~mol} / \mathrm{L}$ sodium cacodylate buffer. Next, the cells were washed three times with $0.1 \mathrm{~mol} / \mathrm{L}$ sodium cacodylate buffer before stained with Hoechst 33258 (Beijing Solarbio Science \& Technology Co., Ltd., China) for $15 \mathrm{~min}$ in the dark.
Finally, an inverted fluorescence microscope (Olympus; BX50-FLA; Japan) was applied to visualize the apoptotic cells.

\section{Immunofluorescence}

Prior to incubation with primary antibody Cleaved caspase-3 (9664; Cell Signaling Technology, Germany) in a diluted concentration of $1: 150$ at $4{ }^{\circ} \mathrm{C}$ overnight, the paraffin-embedded sections need to be deparaffinized, inactivated with $0.3 \%$ hydrogen peroxide for $15 \mathrm{~min}$ and blocked with normal goat serum for 10 mins at room temperature, and then incubated with secondary antibody goat anti-rabbit IgG polyclonal (ab15007; Abcam, United States) for one hour at room temperature and dark place, followed by DAPI staining at room temperature for $5 \mathrm{~min}$ in the dark. Finally, sections were washed and fixed with antifade mounting medium. The optical microscope was used for photographing at $400 \times$ magnification (Leica Microsystems $\mathrm{GmbH}$ ).

H9C2 cells were grown on a laser confocal dish for the specified time, and then fixed with $4 \%$ paraformaldehyde for $15 \mathrm{~min}$, followed by permeabilization $(0.5 \%$ Triton $\mathrm{X}-100$ in $0.1 \mathrm{~mol} / \mathrm{L}$ sodium cacodylate buffer) for 20 min and blocked with normal goat serum for one hour. Later, cells were incubated with primary antibody overnight at $4{ }^{\circ} \mathrm{C}$, followed by incubation with secondary antibody in the dark at room temperature for one hour. After being washed 3 times with PBS, cells were counterstained with DAPI $(5 \mu \mathrm{g} / \mathrm{mL})$ for $30 \mathrm{~min}$. Images were then taken with a confocal microscopy.

\section{Western blot analysis}

Protein samples were prepared and detected according to our previously described methods [26]. The antibodies we used were listed in Table 2.

\section{Data and statistical analysis}

Statistical analyses were performed on GraphPad Prism software 6.0 (San Diego, CA, USA). All results were expressed as the mean $\pm \mathrm{SD}$. Comparisons between two groups were performed with the unpaired two-tailed $t$ test. Multiple comparisons were analyzed using ANOVA followed by Bonferroni-corrected post hoc test. The difference was considered statistically significant when $P<0.05$. The statistical analysis abided the recommendations of the experimental design and analysis in pharmacology [27]. 
Table 2 Primary antibodies used in western blot

\begin{tabular}{ll}
\hline Protein & Primary antibody \\
\hline p-NF-KB & ab97726; Abcam, United States \\
NF-KB & CST8242; Cell Signaling Technology, Germany \\
TNF-a & ab205587; Abcam, United States \\
IL-8 & ABM40268; Abbkine; United States \\
COX2 & ab15191; Abcam; United States \\
p-IKKa/ & CST2697T; Cell Signaling Technology, Germany \\
IKKa & CST2682; Cell Signaling Technology, Germany \\
IKKß & CST8943; Cell Signaling Technology, Germany \\
mTOR & CST2983; Cell Signaling Technology, Germany \\
p-mTOR & CST2971; Cell Signaling Technology; Germany \\
S6K1 & ab9366; Abcam, United States \\
p-S6K1 & ab2571; Abcam, United States \\
GAPDH & ab8245; Abcam, United States \\
rabbit IgG H\&L & ab16284; HRP; Abcam, United States \\
mouse IgG H\&L & ab97250; HRP; Abcam, United States \\
TFEB & 13,372-1-AP; Proteintech; United States \\
\hline
\end{tabular}

\section{Results}

Effects of DHT on cardiac function and structural alteration in DIC zebrafish model and in DIC mice model Two days after DOX exposure, fish exhibited extensive pericardial edema and blood accumulation in tail (Fig. 1a). Microscopic examination revealed that the heart atrium was elongated, and the ventricle collapsed (Fig. 1a). By using a high-speed camera, we calculated the fractional shortening (FS) of the zebrafish hearts. The results showed that FS was dramatically decreased, resulting in reduced erythrocyte circulation within tail blood vessels (Fig. 1a, b, d). Moreover, both heart rate and survival rate were significantly reduced in DOXtreated zebrafish (Fig. 1c, e). DHT treatment protected against cardiotoxicity induced by DOX. It could upregulate FS, promote blood flow in tail vein and rescue the survival rate in DIC zebrafish model.

After four weeks of treatments, all mice were subjected to echocardiography to measure cardiac parameters, including LVEDD, LVESD, EF and FS (Fig. 1f, g). Echocardiography data showed that mice in the DOX group had significantly higher values of LVEDD and LVESD. After treatment with DHT, both of the values decreased. FS was reduced significantly in the DOX group as compared to that of the Saline group, indicative of reduced cardiac contractility. In contrast, FS of mice in the DOX + DHT group was significantly increased compared to mice in the DOX group, suggesting that left ventricular function was improved by DHT treatment. Besides, EF was also upregulated by DHT treatment. Hematoxylin and eosin (H\&E) staining showed that the cardiac tissue was neatly arranged in the Saline group. A high number of infiltrated inflammatory cells and enlargement of the intercellular space could be observed in the DOX group, while treatment with DHT or Rapamycin rescued hearts from inflammatory cell infiltration and cellular injury (Fig. 1h, i). In addition, plasma MDA level was higher and SOD activity was lower in mice of the model group, as compared with those of the Saline group, while DHT treatment reversed the changes (Fig. 1j). Rapamycin had the similar effects to DHT (Fig. 1f-j).

Effects of DHT on the accumulation of macrophages and M1 macrophages activation in DIC mice model and in LPS-stimulated RAW264.7 cells model

It is believed that recruitment of macrophages into the heart plays a pivotal role in the homeostasis of cardiac tissues [28]. Flow cytometry assay was applied to quantify the number of macrophages in cardiac tissue. $\mathrm{CD} 11 \mathrm{~b}+\mathrm{F} 4 / 80+$ were used as biomarkers for macrophages. The results showed that the percentage of macrophages in the DOX group was significantly higher than that of the Saline group, indicating that circulating monocytes were mobilized to inflamed cardiac tissue and differentiated to macrophages in response to DIC. Treatment with DHT or rapamycin inhibited the recruitment of macrophages (Fig. 2a). Activated macrophages can be classified as either M1 (marked by CD86) or M2 (marker by CD206) macrophages. Our data showed a marked increase of M1 macrophages in DOX-treated mice, while treatment with DHT or rapamycin inhibited the activation of M1 macrophages (Fig. 2a). Besides, immunofluorescence assay showed that DHT suppressed the protein expressions of CD86 and F4/80 in mice. There was no difference of distribution of M2 macrophage among the four groups (Fig. 2b). Collectively, these data indicated that DHT exerted antiinflammatory effect in DOX-stimulated mice probably by inhibiting activation of M1 macrophages.

To further investigate the effects of DHT on macrophages, LPS-stimulated RAW264.7 cell model was established. The CCK-8 assay showed that co-treatment of RAW264.7 cells with DHT (10, 50, and $100 \mathrm{nM})$ was nontoxic (Fig. 2c), and DHT could significantly decrease the LPS-induced expressions of TNF- $\alpha$ and IL-1 $\beta$ (Fig. 2d). Besides, immunofluorescence assay showed that DHT suppressed the expression of CD86 under LPSstimulation (Fig. 2e). Flow cytometry assay furtherly proved that DHT could inhibit the activation of M1 macrophages (Fig. 2f). We also found that LPS-stimulation promoted expression and nuclear translocation of p-NF$\mathrm{kB}$ (Fig. 2g). Collectively, the LPS-induced inflammatory responses were inhibited by DHT treatment. These in vitro results confirmed that the anti-inflammatory effect of DHT was partly exerted by suppressing activity of macrophages. 
A
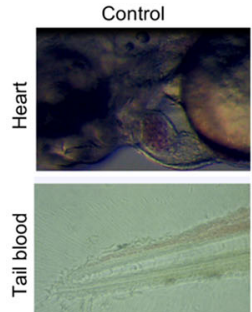

C
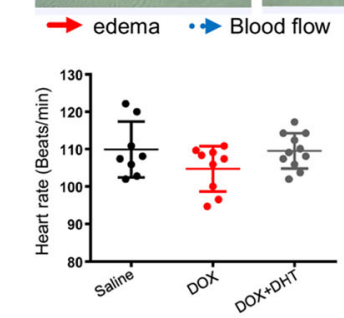

$\mathbf{F}$
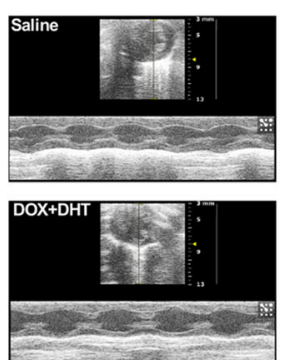

H

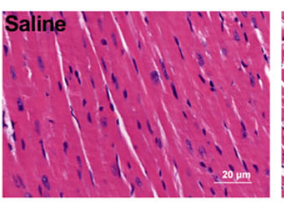

I

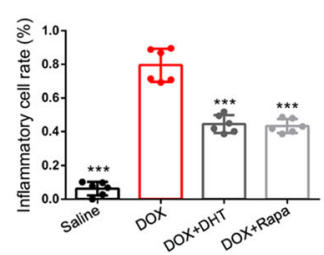

$D O X+D H T$
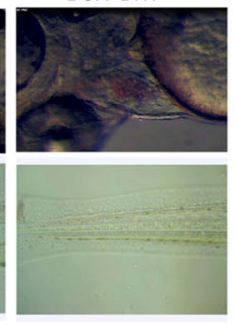

D

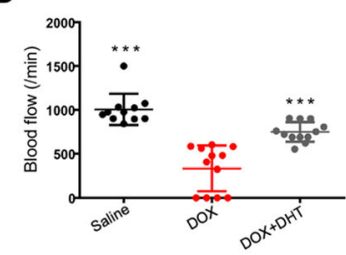

B

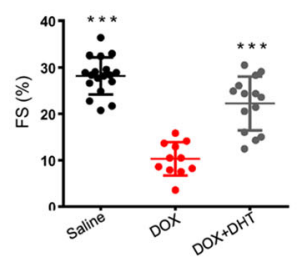

E

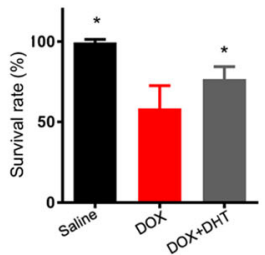

G

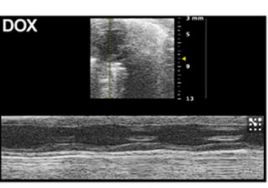

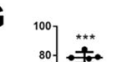
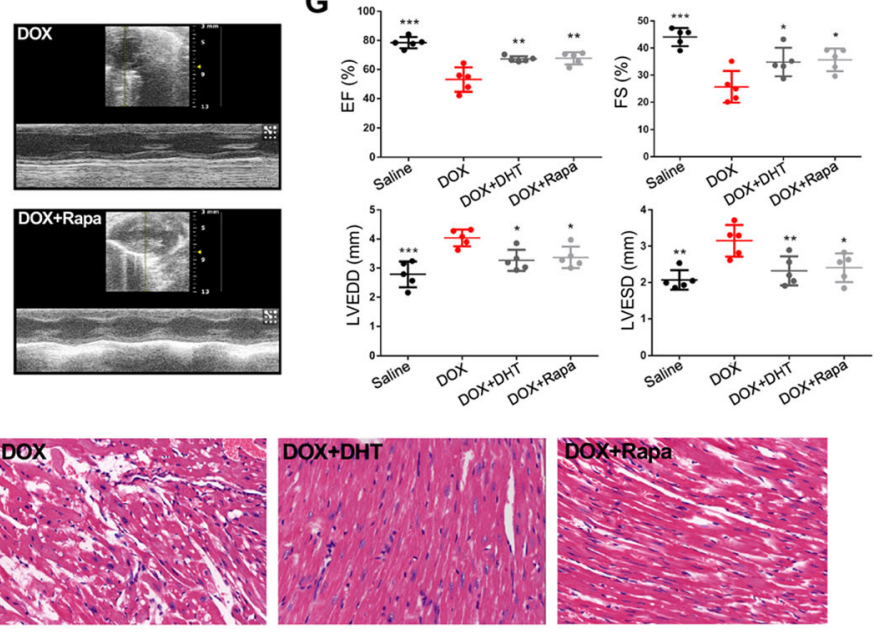

J
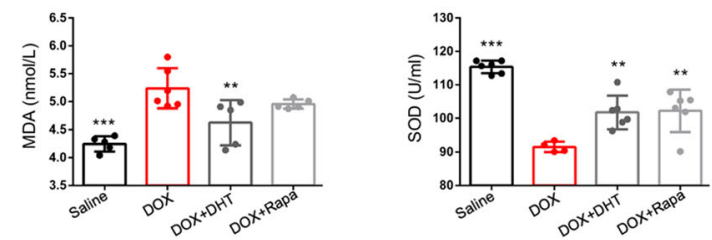

Fig. 1 DHT improved cardiac function and protected against pathological injury in zebrafish and in mice. a Microscopic examination showed that DHT preserved the heart atrium, and protected against extensive pericardial edema and blood accumulation in tail. By using a high-speed camera, results and analysis showed that DHT increased FS values $(\mathbf{b})$ and blood flow $(\mathbf{d})$. DHT could improve heart rate (c) and survival rate (e). f M-mode echocardiography was assessed to detect cardiac function in each group. $\mathbf{g}$ Echocardiography data showed that DHT increased EF\% and FS\%, and decreased LVEDD and LVESD. $\mathbf{h}$ HE staining showed that DHT protected against the structural damage caused by DOX, scale bar = $20 \mu \mathrm{m}$. i Quantification of inflammatory cell infiltration (\%) showed that DHT decreased the inflammatory cell rate. $\mathbf{j}$ Quantification of SOD and MDA levels in plasma. $N \geq 10$ per group in zebrafish; $N \geq 5$ per group in mice. All data were presented as means $\pm S D$ in triplicate. ${ }^{*} p<0.05$, ${ }^{* *} p<0.01,{ }^{* * *} p<0.001$ is significantly different as indicated, for values in the DOX group

\section{Effects of DHT on NF-KB signaling pathway in DIC mice model and in DOX-stimulated $\mathrm{H} 9 \mathrm{C} 2$ cells model}

In mice, western blotting showed that the total NF- $\mathrm{kB}$ expression was unchanged among different groups. However, the expression of activated/phosphorylated NF- $k B$ (p-NF-kB) increased in the heart tissue of DOX group. After treatment with DHT, the expression of pNF- $\mathrm{KB}$ was reduced significantly (Fig. 3a). The expressions of downstream inflammatory genes activated by NF- $\mathrm{kB}$ were further detected. Levels of TNF- $\alpha$, COX2 (Cyclooxygenase-2) and IL- 8 in the DOX group were increased, as compared to the Saline group, whereas DHT 
A
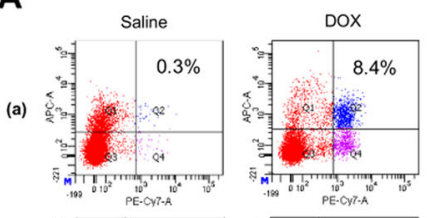

(b)
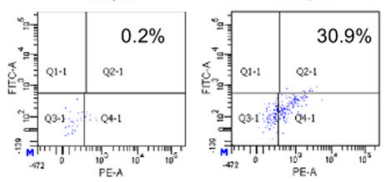
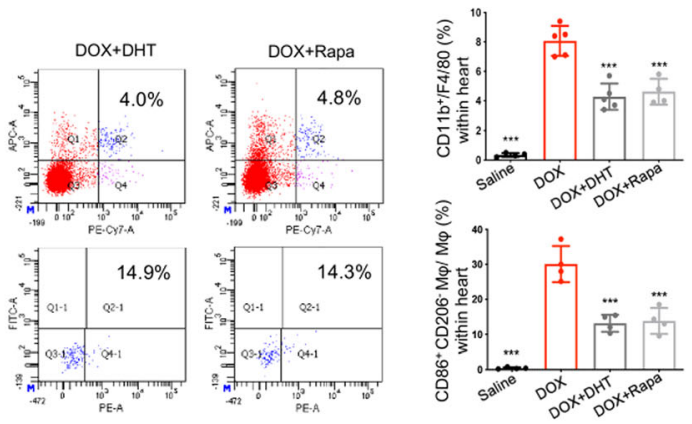

B
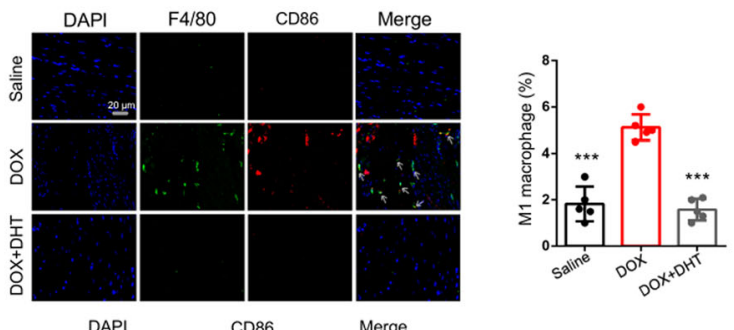

C

E

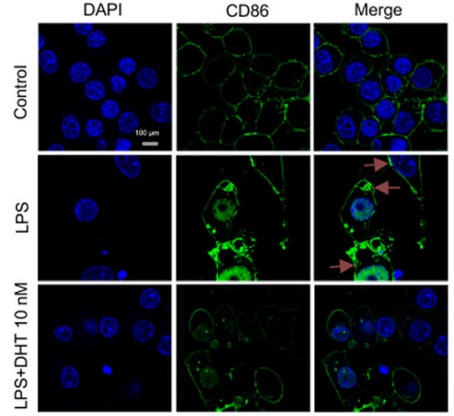

\section{D}
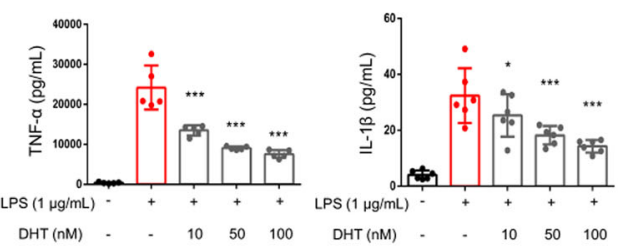

$\mathbf{F}$
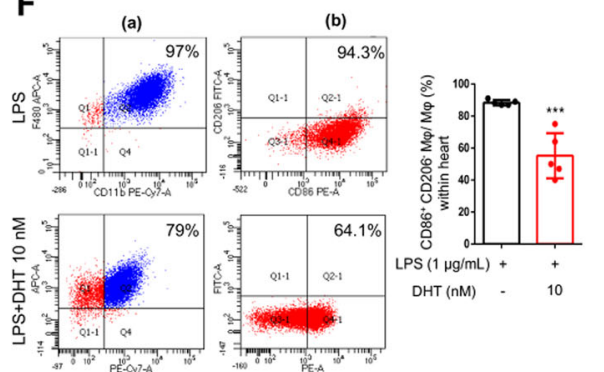

G

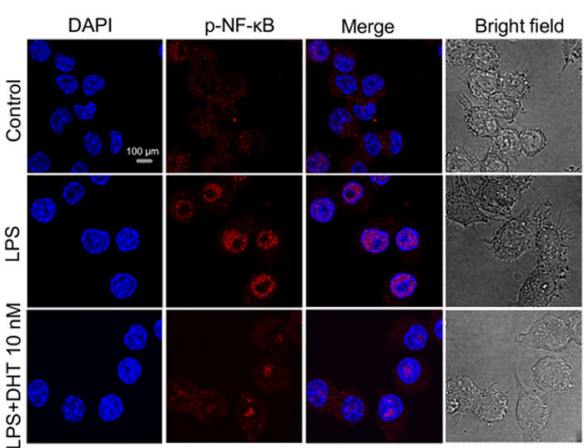

Fig 2 DHT suppressed the activation of M1 macrophages in mice and in RAW264.7 cells. (A) Flow cytometry assay showed that DHT or Rapamycin reduced accumulation of macrophages and activation of M1 macrophages in mice. In (a), X-axis represents PE-Cy7 anti-CD11b and $Y$ axis represents APC anti-F4/80; in (b), X-axis represents PE anti-CD86 and Y-axis represents FITC anti-CD206. N $\geq 3$ per group. ${ }^{*} p<0.05,{ }^{* *} p<0.01$, ${ }^{* * *} p<0.001$ is significantly different as indicated, for values in the DOX group. $\mathbf{b}$ Immunofluorescence assay showed that DHT suppressed the protein expressions of CD86 and F4/80 in mice, scale bar $=20 \mu \mathrm{m}$. N=3 per group. c DHT treatment for $24 \mathrm{~h}$ had no cytotoxic effect on RAW264.7 cells at the dosages of 10,50 and $100 \mathrm{nM}$. $N=6$ per group. $\mathbf{d}$ RAW 264.7 cells were stimulated with LPS (1 $\mu \mathrm{g} / \mathrm{mL})$ in the absence or presence of DHT (10,50 and $100 \mathrm{nM})$ for $24 \mathrm{~h}$ and the releases of TNF- $\mathrm{a}$ and IL-1 $\beta$ in cell supernatants were detected by Elisa assay. $\mathrm{N} \geq 5$ per group. ${ }^{*} p<0.05,{ }^{* * *} p<0.001$ is significantly different as indicated, for values in the LPS group. e The protein expression of CD86 was detected by immunofluorescence assay. DHT suppressed the expression of CD86 in RAW264.7 cells, scale bar $=100 \mu \mathrm{m}$. $\mathrm{N}=12$ per group. $\mathbf{f}$ DHT reduced activation of M1 macrophages in LPS-stimulated RAW264.7 cells. N $\geq 3$ per group. ${ }^{* * *}<0.001$ is significantly different as indicated, for values in the LPS group. $\mathbf{g}$ DHT suppressed the nuclear localization of p-NF-kB in LPS-stimulated RAW264.7 cells, scale bar $=50 \mu m$. $N=20$ per group. All data were presented as means \pm SD in triplicate.

treatment suppressed expressions of these downstream targets (Fig. 3a). To further evaluate the antiinflammatory effects of DHT, the level of TNF- $\alpha$ was assessed. Content of TNF- $\alpha$ in homogenized fresh cardiac tissue was significantly up-regulated in the DOX group whereas treatment with DHT or rapamycin could 
A
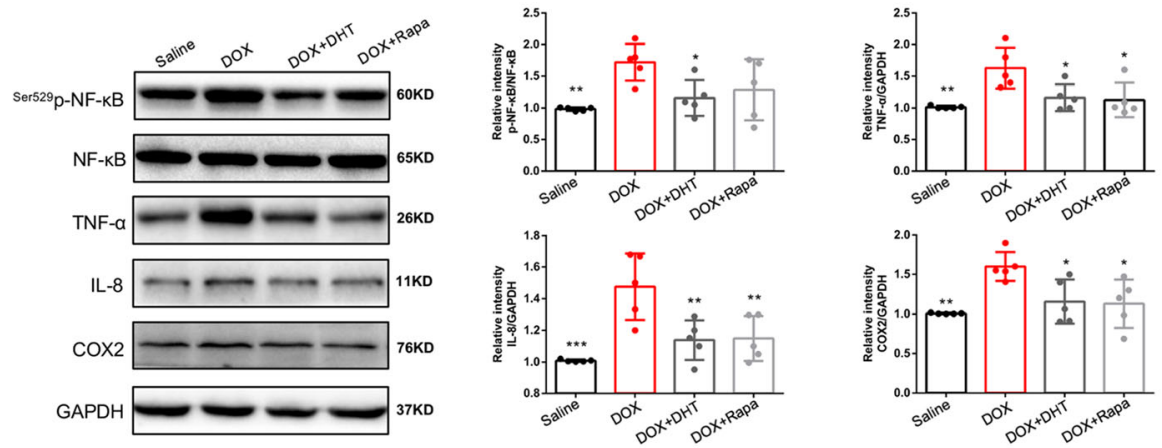

B

C
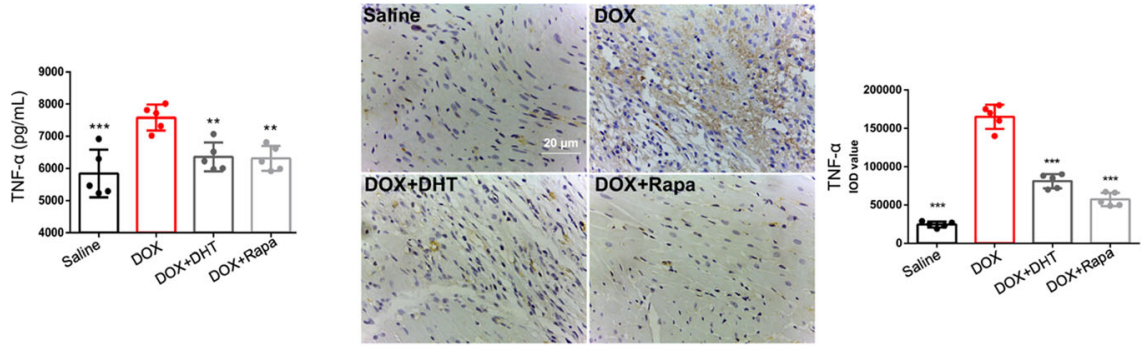

D
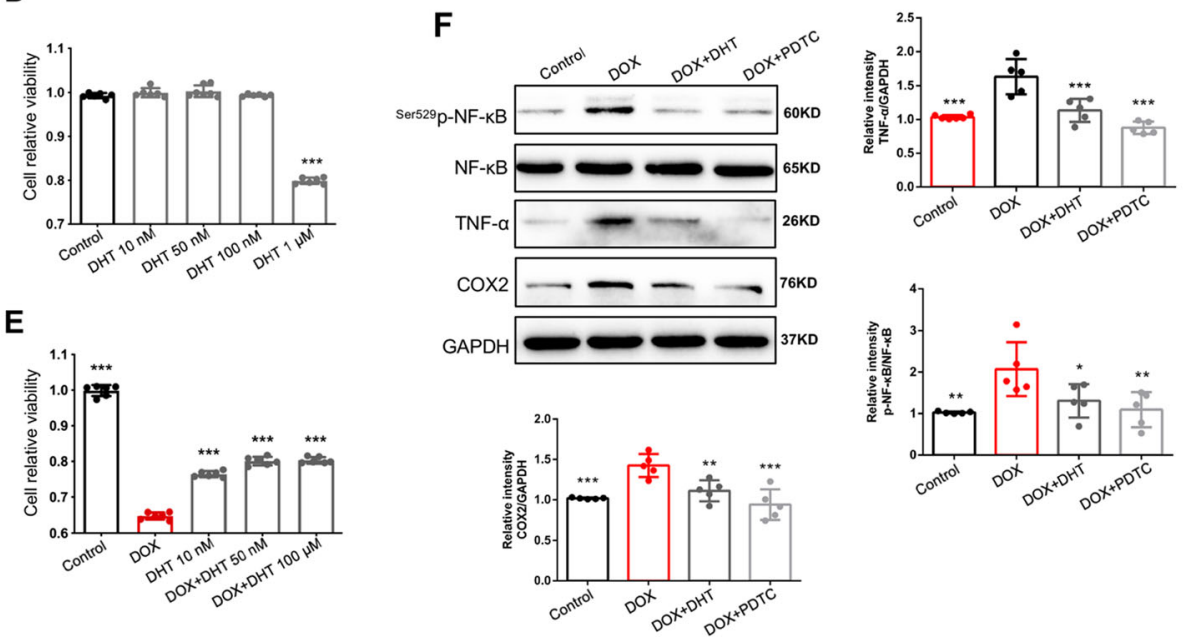

Fig. 3 DHT inhibited the production of pro-inflammatory cytokines mediated by NF-kB in mice and in cardiomyocytes. a Western blotting showed that DHT downregulated the expressions of activated NF-KB, TNF-a, COX2 and IL-8 in mice. N=5 per group. All data were presented as means \pm SD. ${ }^{*} p<0.05,{ }^{* *} p<0.01,{ }^{* * *} p<0.001$ is significantly different as indicated, for values in the DOX group. $\mathbf{b}$ Quantification of TNF-a level in heart tissue. $\mathrm{N}=5$ per group. $\mathbf{c} \| \mathrm{HC}$ and quantitative results showed that DHT or Rapamycin suppressed the expression of TNF-a, scale bar = $20 \mu \mathrm{m} . \mathrm{N}=5$ per group. CCK-8 assay results showed the non-toxic range of concentration (d) and the protective concentrations (e). $N=12$ per group. $\mathbf{f}$ Western blotting showed that DHT downregulated the expressions of activated NF-KB, TNF-a, COX2 in cardiomyocytes. N $=5$ per group. All data were presented as means \pm SD in triplicate. ${ }^{*} p<0.05,{ }^{* *} p<0.01,{ }^{* * *} p<0.001$ is significantly different as indicated, for values in the DOX group

effectively inhibit the expression of TNF- $\alpha$ (Fig. 3b). IHC of TNF- $\alpha$ was assessed and the result was consistent with Elisa result (Fig. 3c).

To further investigate the effects of DHT on NFkB-mediated inflammation in myocardial cells, a DOX-stimulated H9C2 cell model was established [26]. Pre-treatment with $10 \sim 100 \mathrm{nM}$ DHT for $24 \mathrm{~h}$ and co-treatment for $24 \mathrm{~h}$ proved to be effective (Fig. $3 \mathrm{~d}$ and e). Besides, pyrrolidine dithiocarbamate (PDTC) was applied as a positive control drug, due to its direct inhibitory effects on NF- $\kappa B$. Consistent with results in mice, western blotting results in cardiomyocytes showed that the expressions of $\mathrm{p}-\mathrm{NF}-\mathrm{\kappa B}, \mathrm{TNF}-\alpha$ and COX2 were inhibited by DHT treatment, 
suggesting that DHT suppressed inflammation mediated by NF-kB signaling pathway (Fig. 3f).

\section{DHT inhibited inflammation by regulating mTOR-TFEB- NF-KB signaling pathway in DOX-stimulated cardiomyocytes}

Recent studies reported that mTOR accumulation could induce inflammation [29, 30]. In this study, up-regulation of phosphorylated/activated mTOR was discovered in DOX-treated mice (Fig. 4a). The level of phosphorylated S6K, a downstream molecule reflecting the biological activity of mTOR, was also increased in DOX-treated mice (Fig. 4a). In mice treated with DHT, levels of phosphorylated mTOR and S6K were significantly reduced.
Rapamycin showed similar effects in mice with DIC (Fig. 4a).

To further confirm whether DHT inhibited inflammation through targeting mTOR, the mTOR agonist MHY1485 was utilized. Co-incubation with MHY1485 abolished the effects of DHT on expressions of p-NF- $\mathrm{kB}$ and the downstream pro-inflammatory genes, including TNF- $\alpha$ and COX2 (Fig. $4 \mathrm{~b}$ ). To investigate the effects of DHT on TFEB recruitment, H9C2 cells were transfected with a carried TFEB-GFP lentiviral vector (Lv). Results showed that DHT treatment could reduce the cytoplasmic localization and upregulate the nuclear recruitment of TFEB, while co-incubation with MHY1485 abolished this effect, indicating that TFEB is a mediator by which mTOR regulates NF-kB signaling pathway (Fig. 4c).

A
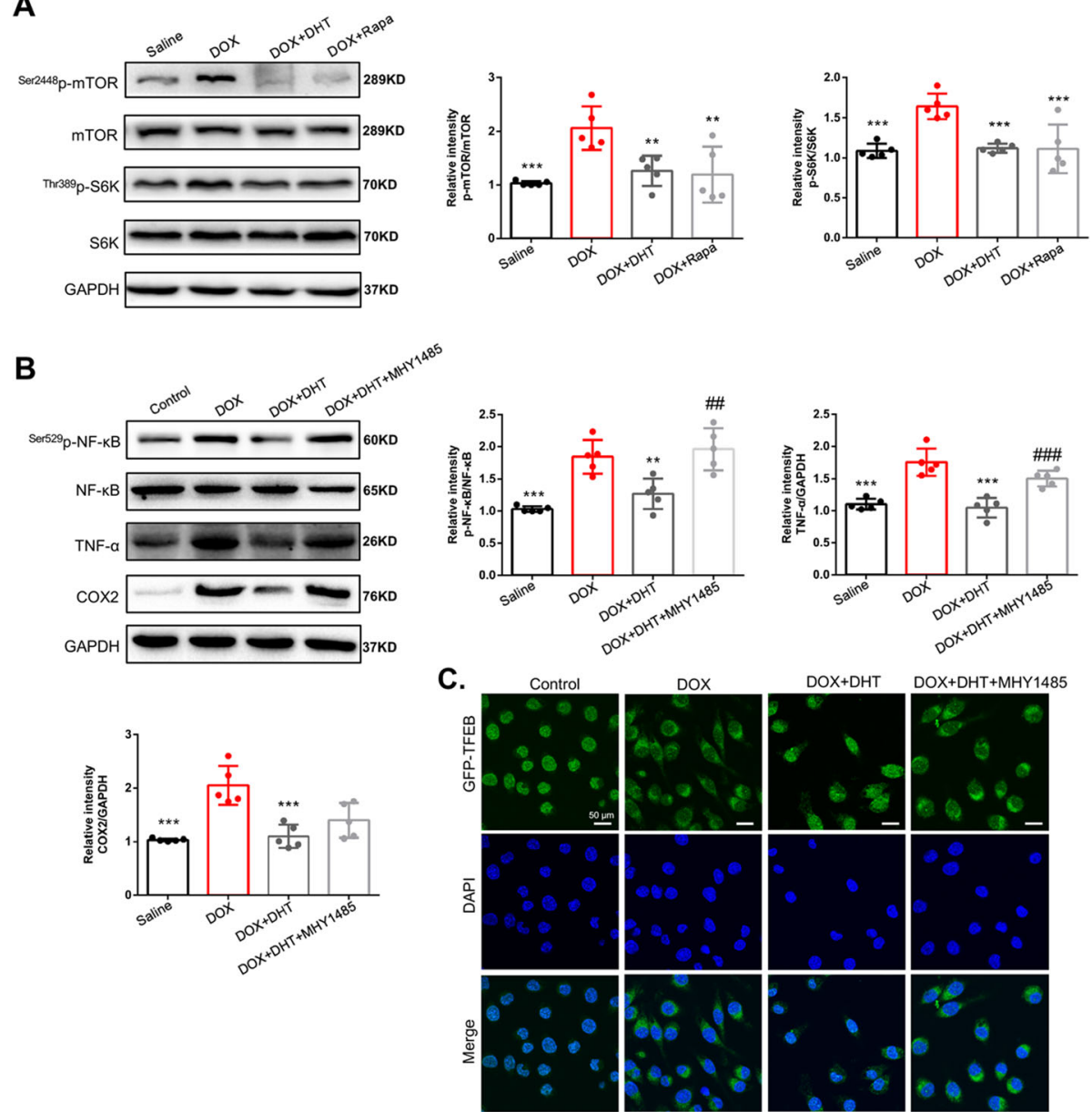

Fig. 4 DHT regulated mTOR-TFEB-NF-KB signaling pathway to ameliorate inflammation in DOX-stimulated cardiomyocytes. a Western blotting showed that DHT or rapamycin downregulated the expressions of phosphorylated mTOR and S6K in mice. $N=5$ per group. All data were presented as means \pm SD in triplicate. ${ }^{*} p<0.05,{ }^{* *} p<0.01,{ }^{* * *} p<0.001$ is significantly different as indicated, for values in the DOX group. $\mathbf{b}$ Western blotting showed that with MHY1485 co-treatment, the effect of DHT on inflammation-related proteins including TNF-a and COX2 was abrogated in cardiomyocytes. $N=5$ per group. All data were presented as means \pm SD in triplicate. ${ }^{*} p<0.05,{ }^{* *} p<0.01,{ }^{* * *} p<0.001$ is significantly different as indicated, for values in the DOX group. ${ }^{\# \#} p<0.01,{ }^{\# \# \#} p<0.001$ is significantly different as indicated, for values in the DOX + DHT group. c Cardiomyocytes were transfected with GFP-TFEB and results showed that co-treatment with MHY1485 abolished the effects DHT on the nuclear localization of TFEB, scale bar $=50 \mu \mathrm{m} . \mathrm{N}=20$ per group 
Collectively, these results demonstrated that DHT ameliorated inflammation partly via mTOR-TFEB-NF-kB signaling pathway.

\section{DHT inhibited TFEB-IKK-NF-KB inflammatory signaling axis in cardiomyocytes}

As compared to Lv-TFEB transfected cells, DOX treatment led to the decreased expression of TFEB in the nucleus (Fig. 5a). As compared to Lv-GFP transfected cells, DOX treatment upregulated the phosphorylation of NF$\kappa B$ (Fig. 5a). It is worth noting that TFEB overexpression significantly reduced $\mathrm{p}-\mathrm{NF}-\mathrm{\kappa} B$ expression and nuclear translocation, as compared to Lv-GFP transfected cells under DOX stimulation (Fig. 5a), furtherly confirming that TFEB could inhibit NF- $\mathrm{kB}$ activation and nuclear translocation. In addition, western blotting showed that phosphorylated level of IKK $\alpha / \beta$ and NF- $k B$ in DOXtreated $\mathrm{H} 9 \mathrm{C} 2$ cells was higher than that of cells without DOX treatment, accompanied by a reduced expression of nuclear TFEB (Fig. 5b-d). TFEB overexpression decreased levels of $\mathrm{p}-\mathrm{IKK} \alpha, \mathrm{p}-\mathrm{IKK} \beta$ and $\mathrm{p}-\mathrm{NF}-\mathrm{kB}$, suggesting that TFEB is an anti-inflammatory factor and may inhibit NF- $\mathrm{kB}$ activity by restraining IKK signaling pathway (Fig. 5b-d).

The effects of DHT on TFEB and IKK $\alpha / \beta$ were further explored. As shown in Fig. 5b, c and d, DHT treatment promoted nuclear localization of TFEB under DOXstimulation. Meanwhile, after treatment with DHT, expressions of $\mathrm{p}-\mathrm{IKK} \alpha / \beta$ and $\mathrm{p}-\mathrm{NF}-\mathrm{kB}$ were reduced (Fig. $5 \mathrm{~b}-\mathrm{d}$ ). Furthermore, DHT treatment inhibited nuclear localization of $\mathrm{p}-\mathrm{NF}-\mathrm{kB}$ (Fig. 5e). Inhibiting TFEB through application of mTOR agonist could abolish the effects of DHT on p-NF-kB (Fig. 4). Taken together, these data demonstrated that DHT inhibited NF-kB transcriptional activity via TFEB-IKK signaling pathway.

\section{DHT inhibited apoptosis of cardiomyocytes to facilitate the anti-inflammation effect}

It is widely accepted that apoptosis is a potent inducer of inflammation [31]. The anti-apoptotic effects of DHT were further investigated. In vivo results showed that Cleaved caspase-3 expression in the left ventricle of heart was significantly increased in mice with DOX treatment, relative to mice in the Saline group (Fig. 6a). In mice treated with DHT or rapamycin, the expressions of Cleaved-Caspase 3 were reduced. Western blotting showed that DHT could also reduce expression of Bax and increase expression of $\mathrm{Bcl}-2$ in mice treated with DOX, illustrating that DHT had anti-apoptotic effect (Fig. 6b).

Consistent with the in vivo experiment, in vitro study demonstrated that DHT significantly reduced the apoptotic rate, and regulated expressions of $\mathrm{Bcl}-2$ and $\mathrm{Bax}$ in DOX-stimulated $\mathrm{H} 9 \mathrm{C} 2$ cells (Fig. 6c, d). Interestingly, co-incubation with MHY1485 abolished the protective effects of DHT against apoptosis (Fig. 6c, d), indicating that the anti-apoptotic effect of DHT was partially mediated through mTOR pathway.

\section{Discussion}

In the present study, in vivo and in vitro experiments were conducted to explore the protective effects of DHT against DIC and its underlying anti-inflammation mechanisms. The main findings are as follows: (1) DHT could improve cardiac functions evidenced by inhibiting the activation of M1 macrophages and excessive release of pro-inflammatory cytokines both in vivo (mouse and zebrafish) and in vitro (H9C2 and RAW264.7); (2) DHT could inhibit NF- $\kappa B$-mediated inflammatory response via mTOR-TFEB pathway; (3) The TFEB-IKK-NF- $\kappa B$ axis plays a vital role in regulating DIC-related inflammation.

A growing body of evidence supports that DOX, as an effective chemotherapy drug, is a double-edged sword [32]. Indeed, irreversible injury to nontarget tissues often complicates cancer care by limiting therapeutic dosages of DOX and lowering the quality of patients' life during and after DOX treatment [33]. Particularly, the heart is a preferential target of DOX and cardiotoxicity is one of the most severe side effects induced by DOX. Alterations in the administration of the drug, applications of liposomal DOX, and assistants with cardio-protective agents are the strategies that have been applied for the prevention or alleviation of cardiotoxicity. However, drugs that are commonly adopted to reduce DIC often have adverse effects. It is of great importance to explore novel therapeutic strategies with fewer side effects. A correlation between inflammation and adverse cardiovascular outcomes in DOX induced toxicity has been documented [34, 35]. Elevation of pro-inflammatory cytokines has been linked with worse DIC outcome in multiple studies [5]. Application of Chinese herbs that are able to mitigate inflammation without compromising the anti-tumor effect is of particular interest for the treatment of DIC.

Salvia miltiorrhiza Bunge is a well-known traditional herb with a long history of clinical application for the treatment of cardiovascular diseases [36, 37]. Its active ingredients have been widely investigated since last century [38]. For a long time, investigations into the bioactivities of DHT were mainly focused on cytotoxicity to various tumor cells $[16,39]$. Emerged evidence pointed out that DHT has potential efficacy for curing cardiovascular diseases [40-42], however its anti-DIC effect, either in vivo or in vitro, has not been elucidated yet. The results of our study demonstrated that DHT treatment improved heart function in a DIC zebrafish model and in a DIC mouse model. It's reported that DHT could 
A

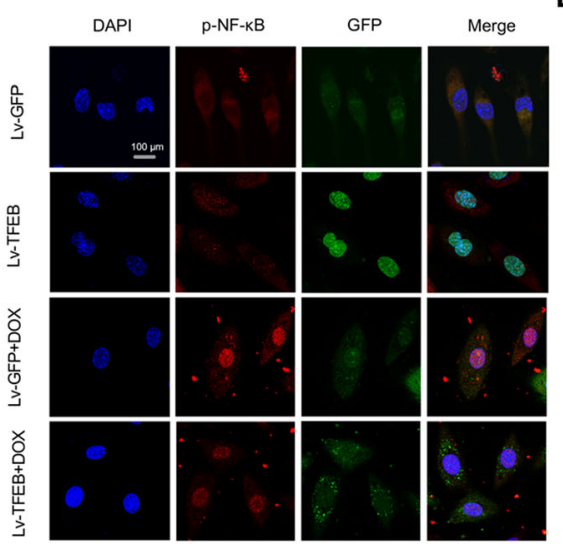

C

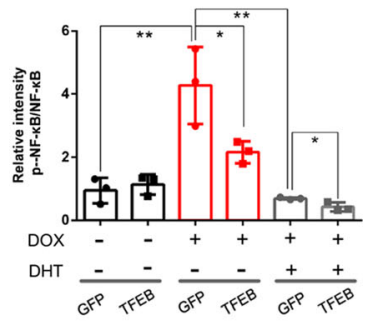

D
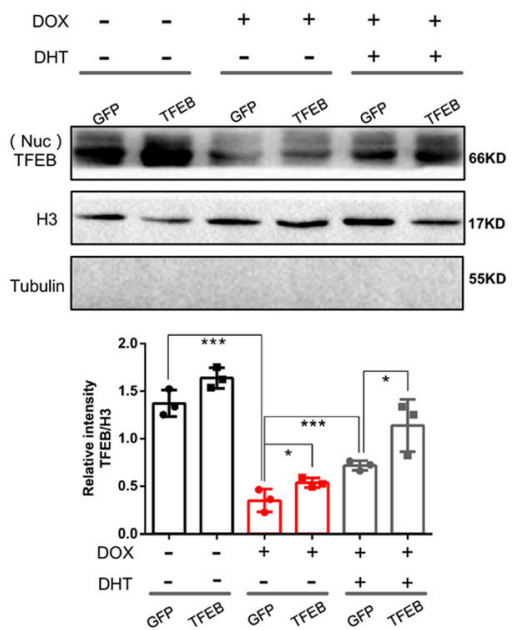

E

B

$\mathrm{DOX}-\quad-\quad+\quad+\quad+\quad+$

DHT
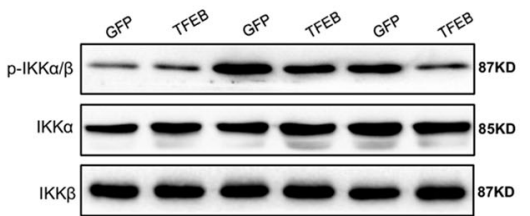

$\mathrm{KK}$
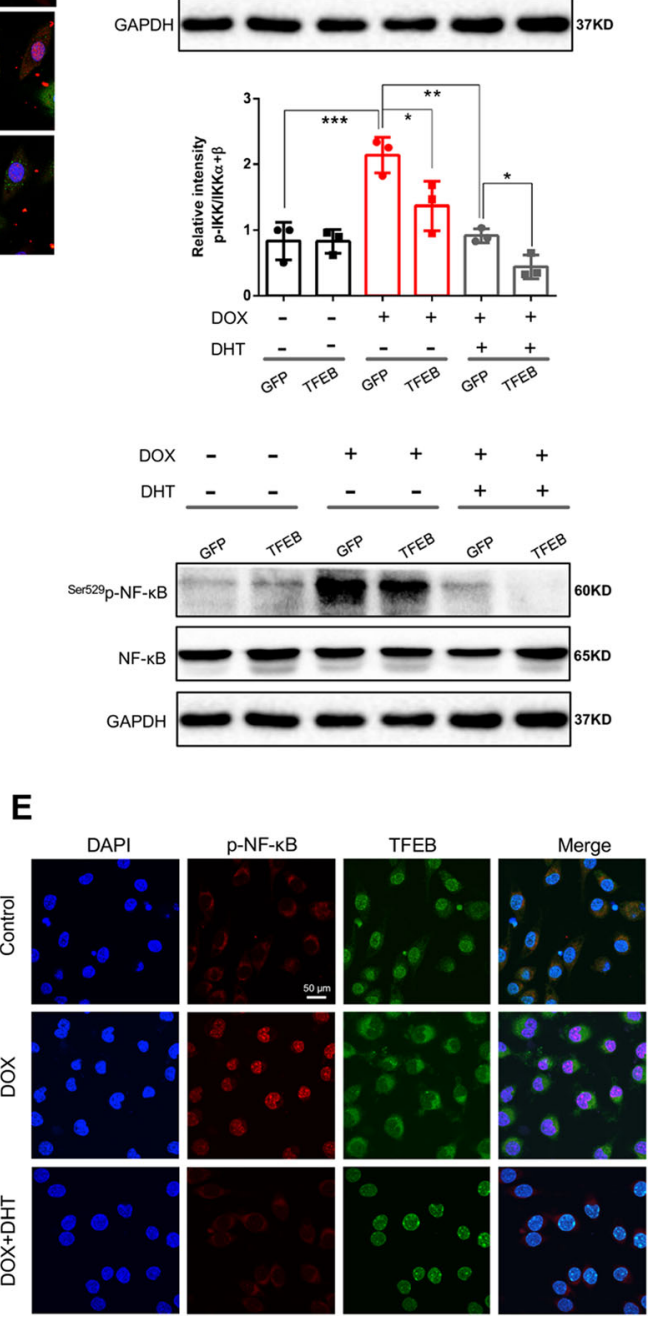

Fig. 5 DHT inhibited NF-KB transcriptional activity via TFEB-IKK signaling pathway. a Cardiomyocytes were transfected with Lentiviral vector (Lv) carrying GFP-TFEB to monitor the location of TFEB. LV-GFP transfected cells were set as a negative control. Representative GFP fluorescence and immunofluorescence staining of p-NF-KB were shown under different treatment, scale bar $=100 \mu \mathrm{m}$. $\mathrm{N}=20$ per group. $\mathbf{b}$, $\mathbf{c}$, $\mathbf{d}$ Western blotting showed that DOX reduced the nuclear expression of TFEB and increased phosphorylated levels of IKKa/ $\beta$ and NF-KB, while DHT up-regulated the nuclear expression of TFEB. In particular, TFEB overexpression or DHT treatment down-regulated phosphorylated levels of IKKa/ $\beta$ and NF-KB. $N=3$ per group. e Representative GFP-TFEB fluorescence and immunofluorescence staining of p-NF-KB under different treatment, scale bar $=50 \mu \mathrm{m}$. $N=20$ per group. All data were presented as means \pm SD in triplicate. ${ }^{* *} p<0.05,{ }^{* *} p<0.01,{ }^{* * *} p<0.001$

play a therapeutic role in various inflammatory diseases, including atherosclerosis, allergic inflammation and colitis [43, 44]. Besides, DHT could modulate immune cell function, such as suppression of the release of cytokines [45]. To date, there have been no researches on the antiinflammatory of DHT during application of DOX. Recruitment and activation of M1 macrophages have been reported to play a major role in DIC-related inflammation [34, 35]. Our in vivo and in vitro results showed that DHT could suppress accumulation of macrophages and activation of M1 macrophages under DOXstimulation. Although it is still unclear as to the origin of heart macrophages, recent studies have suggested that these macrophages are derived from either the 
A
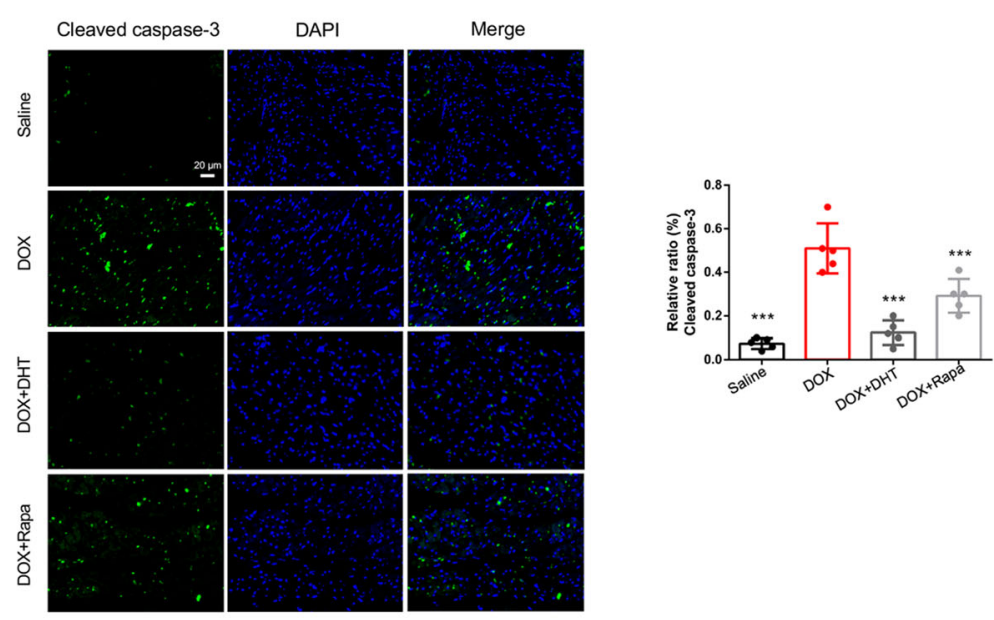

B
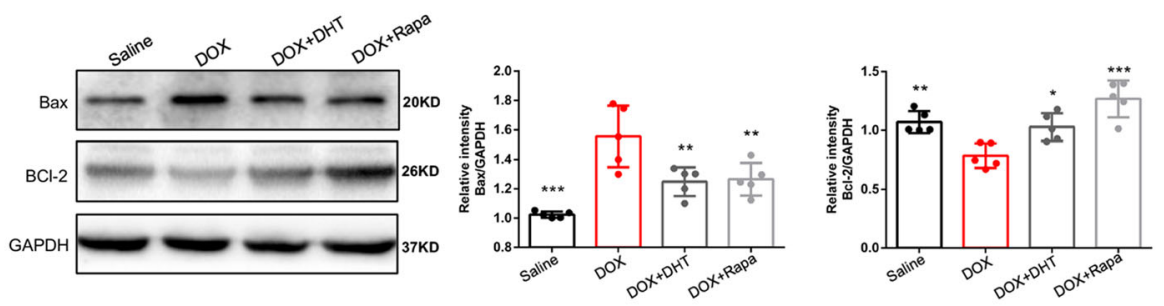

C
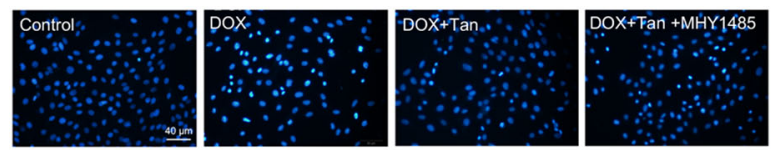

\section{D}
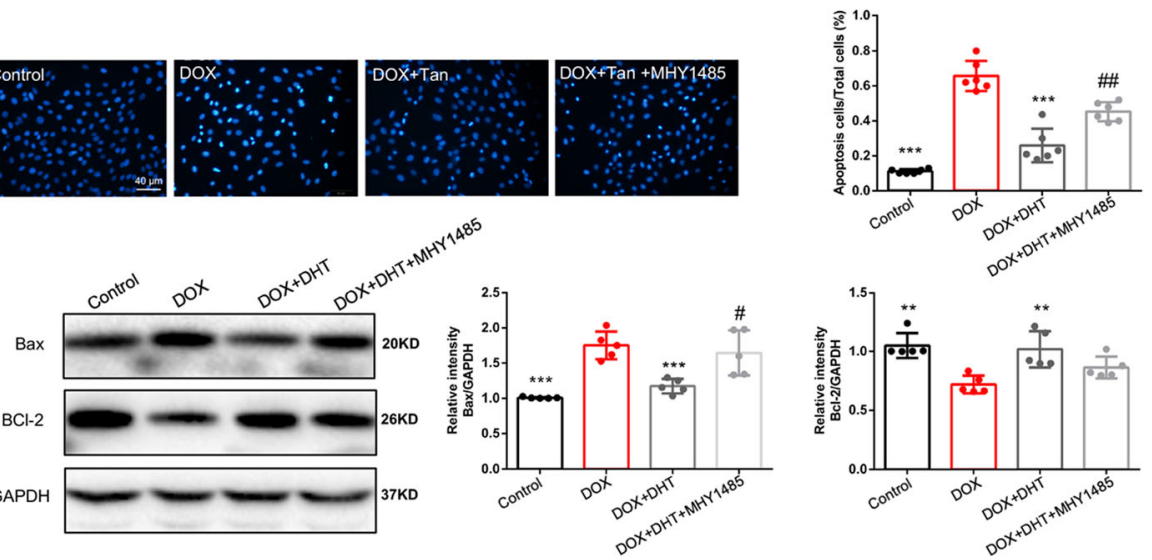

Fig. 6 DHT inhibited apoptosis to facilitate the anti-inflammation effect. a The representative photomicrographs of a left ventricle section of the heart showed the expression of Cleaved caspase-3 under different treatment, scale bar $=20 \mu \mathrm{m} . \mathrm{N}=5$ per group. $\mathbf{b}$ Western blots detected the expressions of Bax and $\mathrm{Bcl}-2$ proteins in heart tissues. $\mathrm{N}=5$ per group. $\mathbf{c}$ The results of Hoechst staining under different treatment, scale bar $=$ $40 \mu \mathrm{m}$. $\mathrm{N}=12$ per group. $\mathbf{d}$ Western blots assessed the expressions of Bax and Bcl-2 proteins in H9C2 cells. $\mathrm{N}=5$ per group. All data were presented as means \pm SD in triplicate. ${ }^{*} p<0.05,{ }^{* *} p<0.01,{ }^{* * *} p<0.001$ is significantly different as indicated, for values in the DOX group. ${ }^{\#} p<0.05$, ${ }^{\# \#} p<0.01$ is significantly different as indicated, for values in the DOX $+D H T$ group

proliferation of resident macrophages or the differentiation of blood monocytes [46]. The in vitro results showed that expression of NF- $\mathrm{kB}$ and secretion of proinflammatory cytokines by macrophages were also inhibited by DHT. These data demonstrated that DHT could suppress inflammation by inhibiting activation of macrophages. Although pro-inflammatory cytokines are generally produced by activated macrophages, myocardial cells can also produce inflammatory agents through NF$\kappa \mathrm{B}$-dependent pathway under pathological conditions.
It's noteworthy that NF- $\mathrm{kB}$-mediated inflammatory response has been demonstrated as a pivotal pathway in DIC model [47, 48]. The involvement of proinflammatory cytokines driven by the activation of NF$\mathrm{\kappa B}$ can lead to the severe myocardial injury manifested by the dramatic reduction of the heart function $[6,49$, 50]. Herein, the NF- $\mathrm{kB}$ pathway is believed to be one of the most attractive targets for DIC patients [48]. In current study, both in vivo and in vitro data showed that DHT suppressed cardiac levels of activated NF-kB as 
well as downstream inflammatory genes, including TNF$\alpha$, IL- 8 and COX2 under DOX stimulation. The effect of DHT on the upstream regulative pathway was further investigated.

The mTOR protein is a serine/threonine kinase that regulates a variety of cellular functions. Update studies suggest that it is also an important regulator of inflammation responses. A number of studies have indicated that pharmacological inhibition of mTOR can provide anti-inflammatory protection $[20,30,51]$. Rapamycin is a specific inhibitor of mTOR and was applied as positive control drug in this study. Intriguingly, rapamycin dramatically improved cardiac functions and inhibited inflammatory response in DIC models. DHT had similar inhibitory effect on mTOR as rapamycin, providing evidence that mTOR is a potential pharmacological target of inflammation response in DIC. Previous study reported that mTOR inhibitors augmented the antiinflammatory activities of regulatory $\mathrm{T}$ cells and reduced the production of pro-inflammatory cytokines by macrophages [52]. In this study, we focused primarily on the inflammatory regulatory effects and mechanisms of mTOR signaling pathway in cardiomyocytes. The mTOR agonist, MHY1485, was applied to DOX-stimulated H9C2 cells. After co-incubation with MHY1485, the effects of DHT on NF-kB, TNF- $\alpha$, COX2 and nuclear TFEB were abrogated, suggesting that the protective mechanism of DHT on inflammatory response is mainly mediated by mTOR-NF- $\mathrm{BB}$ signaling pathway, moreover, TFEB plays pivotal roles in this signaling pathway.

TFEB has been recently identified as serving critical and diverse roles in immune systems [8]. Then, to verify how the TFEB participates in mTOR-NF-kB pathway, loss/gain of the function of TFEB were performed. We found that DOX treatment reduced the expression of nuclear TFEB, and up-regulated phosphorylation of $I K K \alpha / \beta$ and NF- $\kappa B$, suggesting that there might be a link between TFEB and NF- $\mathrm{kB}$ activation. When $\mathrm{H} 9 \mathrm{C} 2$ cells were transfected with lentiviral vector carrying GFPTFEB, TFEB overexpression downregulated the expressions of activated $I K K \alpha / \beta$ and NF- $\kappa B$, further indicating that the IKK-NF- $\mathrm{KB}$ signaling axis is directly inhibited by TFEB. Targeting TFEB using pharmacological agents may, therefore, hold great promise against cardiac inflammatory complications. Intriguingly, DHT treatment promoted nuclear localization of TFEB and downregulated the expressions of $\mathrm{p}-\mathrm{IKK} \alpha / \beta$ and $\mathrm{p}-\mathrm{NF}-\mathrm{\kappa} \mathrm{B}$, while inhibiting TFEB through application of mTOR agonist could abolish the effects of DHT on p-NF-kB. These data demonstrated that DHT inhibited NF- $\mathrm{kB}$ transcriptional activity via TFEB-IKK signaling pathway. Taken together, our data offered the evidence that DHT inhibited NF-kB-mediated inflammatory response through mTOR-TFEB-IKK signaling pathway.
In the present study, we also investigated the antiapoptotic effects of DHT. Though apoptotic cells could trigger or potentiate inflammation, the underlying mechanism remains to be clarified [31]. In consistent with previous reports [53-55], we found that DOX could induce apoptosis and DHT has anti-apoptotic effects. We further explored the role of mTOR in apoptosis [56]. Intriguingly, co-incubation of cells with mTOR agonist abolished the inhibitory effects of DHT on apoptosis, suggesting that DHT exerted anti-apoptosis effects via mTOR signaling pathway. The mTOR signaling may serve as the co-regulator of both inflammatory and apoptotic pathway. The underlying molecular mechanism warrants further investigation.

\section{Conclusion}

For the first time, our research demonstrated TFEBIKK-NF- $\mathrm{KB}$ inflammatory signaling axis as a novel therapeutic pathway for DIC. DHT can be applied as a potential agent for the anti-inflammation treatment of DIC via mTOR-TFEB-NF- $k B$ signaling pathway. This study provides the possibility of adjuvant therapies that protect the heart as well as improving antitumor effects. In addition, it will be informative to explore whether DHT's cardioprotective effects could extend to other modes of heart injury, such as ischemia or other cardiotoxic chemotherapies.

\section{Abbreviations \\ CMC: Carboxymethylcellulose; COX2: Cyclooxygenase-2; \\ DAB: Diaminobenzidine; DHT: Dihydrotanshinone I; DIC: Doxorubicin-induced cardiotoxicity; DMEM: Dulbecco's Modification of Eagle's Medium; DMSO: Dimethyl sulfoxide; DOX: Doxorubicin; dpf: Day postfertilization; EF: Ejection fraction; FS: Fraction shortening; HE: Hematoxylin-Eosin; IKB: Inhibitor of NF-KB; IKKs: IKB kinases; IL-8: Interleukin 8; LVEDD: Left ventricular end-diastolic dimension; LVEDV: Left ventricular end-diastolic vol- ume; LVESD: Left ventricular end-systolic dimension; LVESV: Left ventricular end-systolic volume; MDA: Malondialdehyde; mTOR: Mammalian target of rapamycin; NF-KB: Nuclear factor-KB; PBS: Phosphate buffer saline; PDTC: Pyrrolidine dithiocarbamate; Rapa: Rapamycin; SOD: Superoxide dismutase; SPF: Specific Pathogen Free; TCM: Traditional Chinese medicine; TFEB: Transcription factor EB; TNF-a: Tumor necrosis factor-a}

\section{Acknowledgements}

Not applicable.

\section{Authors' contributions}

XP. Wang performed the research, analyzed the data, and wrote the manuscript. QY. Wang wrote and revised the manuscript. WL. Li, Q. Zhang and DQ. Guo contributed to in vitro experiments. WJ. Lu, XQ. Sun and YY. Jiang contributed to echocardiography. Y. Wang, W. Wang and C. Li funded the research, revised the manuscript, and approved the submission of this manuscript. The authors read and approved the final manuscript.

\section{Funding}

This work was supported by the National Natural Science Foundation of China [grant numbers 81822049, 81673712 and 81673802], Major New Drug Creation of Ministry of Science and Technology [grant number

2019ZX09201004-001-011], the Fok Ying Tung Education Foundation [grant number 151044], the Beijing Nova Program [grant number

Z171100001117028] and Talent Young Scientist of China Association for Science and Technology [grant numbers CACM-2017-QNRC2-C13 and CACM-2018-QNRC2-C07]. 


\section{Availability of data and materials}

The datasets used and/or analysed during the current study are available from the corresponding author on reasonable request.

\section{Ethics approval and consent to participate}

All animal procedures were carried out in accordance with the Guide for the Care and Use of Laboratory Animals (NIH Publications No.85-23) and with Beijing University of Chinese Medicine Animal Care Committee (approve code BUCM-4-2018101504-4068)

\section{Consent for publication}

Not applicable.

\section{Competing interests}

The authors declare that they have no competing interests.

\section{Author details}

${ }^{1}$ School of Life Science, Beijing University of Chinese Medicine, Beijing 100029, China. ${ }^{2}$ School of Traditional Chinese Medicine, Beijing University of Chinese Medicine, Beijing 100029, China. ${ }^{3}$ School of Chinese Materia Medica, Beijing University of Chinese Medicine, Beijing 100029, China.

\section{Received: 24 March 2020 Accepted: 11 May 2020}

Published online: 24 May 2020

\section{References}

1. Damiani RM, Moura DJ, Viau CM, Caceres RA, Henriques JAP, Saffi J. Pathways of cardiac toxicity: comparison between chemotherapeutic drugs doxorubicin and mitoxantrone. Arch Toxicol. 2016:90(9):2063-76.

2. Brewster DH, Clark D, Hopkins L, Bauer J, Wild SH, Edgar AB, Wallace WH. Subsequent hospitalisation experience of 5-year survivors of childhood, adolescent, and young adult cancer in Scotland: a population based, retrospective cohort study. Br J Cancer. 2014:110(5):1342-50.

3. Geisberg CA, Sawyer DB. Mechanisms of anthracycline cardiotoxicity and strategies to decrease cardiac damage. Curr Hypertens Rep. 2010;12(6):40410.

4. Briasoulis A, Androulakis E, Christophides T, Tousoulis D. The role of inflammation and cell death in the pathogenesis, progression and treatment of heart failure. Heart Fail Rev. 2016;21(2):169-76.

5. Akolkar G, da Silva DD, Ayyappan P, Bagchi AK, Jassal DS, Salemi VMC, Irigoyen MC, De Angelis K, Singal PK. Vitamin C mitigates oxidative/ nitrosative stress and inflammation in doxorubicin-induced cardiomyopathy. Am J Physiol Heart Circ Physiol. 2017;313(4):H795-809.

6. Andreadou I, Mikros E, loannidis K, Sigala F, Naka K, Kostidis S, Farmakis D, Tenta R, Kavantzas N, Bibli Sl, et al. Oleuropein prevents doxorubicininduced cardiomyopathy interfering with signaling molecules and cardiomyocyte metabolism. J Mol Cell Cardiol. 2014;69:4-16.

7. Wu J, Sun C, Wang R, Li J, Zhou M, Yan M, Xue X, Wang C. Cardioprotective effect of paeonol against epirubicin-induced heart injury via regulating miR1 and PI3K/AKT pathway. Chem Biol Interact. 2018:286:17-25.

8. Brady OA, Martina JA, Puertollano R. Emerging roles for TFEB in the immune response and inflammation. Autophagy. 2018;14(2):181-9.

9. Napolitano G, Ballabio A. TFEB at a glance. J Cell Sci. 2016:129(13):2475-81.

10. Song W, Zhang CL, Gou L, He L, Gong YY, Qu D, Zhao L, Jin N, Chan TF, Wang $L$, et al. Endothelial TFEB (transcription factor EB) restrains IKK (IkappaB kinase)-p65 pathway to attenuate vascular inflammation in diabetic db/db mice. Arterioscler Thromb Vasc Biol. 2019:39(4):719-30.

11. Gao H, Huang L, Ding F, Yang K, Feng Y, Tang H, Xu Q-M, Feng J, Yang S. Simultaneous purification of dihydrotanshinone, tanshinone $\mathrm{I}$, cryptotanshinone, and tanshinone IIA from Salvia miltiorrhiza and their antiinflammatory activities investigation. Sci Rep. 2018;8(1):8460

12. Gao H, Liu X, Sun W, Kang N, Liu Y, Yang S, Xu QM, Wang C, Chen X. Total tanshinones exhibits anti-inflammatory effects through blocking TLR4 dimerization via the MyD88 pathway. Cell Death Dis. 2017:8(8):e3004.

13. Wang $X$, Yang Y, Liu X, Gao X. Pharmacological properties of tanshinones, the natural products from Salvia miltiorrhiza. Adv Pharmacol. 2020:87:43-70.

14. Yuan R, Huang L, Du LJ, Feng JF, Li J, Luo YY, Xu QM, Yang SL, Gao H, Feng YL. Dihydrotanshinone exhibits an anti-inflammatory effect in vitro and in vivo through blocking TLR4 dimerization. Pharmacol Res. 2019;142:102-14.
15. Yu L, Qian J. Dihydrotanshinone I alleviates spinal cord injury via suppressing inflammatory response, Oxidative Stress and Apoptosis in Rats. Med Sci Monit. 2020;26:e920738.

16. Kim SL, Choi HS, Kim JH, Jeong DK, Kim KS, Lee DS. Dihydrotanshinoneinduced NOX5 activation inhibits breast Cancer stem cell through the ROS/ Stat3 signaling pathway. Oxid Med Cell Longev. 2019;2019:9296439.

17. Lee CH, Rodeo SA, Fortier LA, Lu C, Erisken C, Mao JJ. Protein-releasing polymeric scaffolds induce fibrochondrocytic differentiation of endogenous cells for knee meniscus regeneration in sheep. Sci Transl Med. 2014;6(266): 266 ra171.

18. Li DL, Wang ZV, Ding G, Tan W, Luo X, Criollo A, Xie M, Jiang N, May H, Kyrychenko V, et al. Doxorubicin blocks Cardiomyocyte Autophagic flux by inhibiting lysosome acidification. Circulation. 2016;133(17):1668-87.

19. Flynn JM, O'Leary MN, Zambataro CA, Academia EC, Presley MP, Garrett BJ, Zykovich A, Mooney SD, Strong R, Rosen CJ, et al. Late-life rapamycin treatment reverses age-related heart dysfunction. Aging Cell. 2013;12(5):851-62.

20. Xu L, Brink M. mTOR, cardiomyocytes and inflammation in cardiac hypertrophy. Biochim Biophys Acta. 2016;1863(7 Pt B):1894-903.

21. Zhao W, Li C, Gao H, Wu Q, Shi J, Chen X. Dihydrotanshinone I attenuates atherosclerosis in ApoE-deficient mice: role of NOX4/NF-KB mediated Lectin-like oxidized LDL Receptor-1 (LOX-1) of the endothelium. Front Pharmacol. 2016;7:418.

22. Belenchia AM, Gavini MP, Toedebusch RG, DeMarco VG, Pulakat L. Comparison of cardiac miRNA Transcriptomes induced by diabetes and Rapamycin treatment and identification of a Rapamycinassociated cardiac MicroRNA signature. Oxid Med Cell Longev. 2018; 2018:8364608.

23. Jung S-B, Choi MJ, Ryu D, Yi H-S, Lee SE, Chang JY, Chung HK, Kim YK, Kang $\mathrm{SG}$, Lee $\mathrm{JH}$, et al. Reduced oxidative capacity in macrophages results in systemic insulin resistance. Nat Commun. 2018;9(1):1551.

24. Ibricevic A, Guntsen SP, Zhang K, Shrestha R, Liu Y, Sun JY, Welch MJ, Wooley KL, Brody SL. PEGylation of cationic, shell-crosslinked-knedel-like nanoparticles modulates inflammation and enhances cellular uptake in the lung. Nanomedicine. 2013;9(7):912-22.

25. Vermaelen K, Pauwels R. Accurate and simple discrimination of mouse pulmonary dendritic cell and macrophage populations by flow cytometry: methodology and new insights. Cytometry A. 2004;61(2): $170-7$.

26. Wang X, Li C, Wang Q, Li W, Guo D, Zhang X, Shao M, Chen X, Ma L, Zhang Q, et al. Tanshinone IIA Restores Dynamic Balance of Autophagosome/ Autolysosome in Doxorubicin-Induced Cardiotoxicity via Targeting Beclin1/ LAMP1. Cancers (Basel). 2019;11(7):910.

27. Curtis MJ, Bond RA, Spina D, Ahluwalia A, Alexander SP, Giembycz MA, Gilchrist A, Hoyer D, Insel PA, Izzo AA, et al. Experimental design and analysis and their reporting: new guidance for publication in $\mathrm{BJP} . \mathrm{Br} J$ Pharmacol. 2015;172(14):3461-71

28. Ma Y, Mouton AJ, Lindsey ML. Cardiac macrophage biology in the steadystate heart, the aging heart, and following myocardial infarction. Transl Res. 2018;191:15-28.

29. Li J, Kim SG, Blenis J. Rapamycin: one drug, many effects. Cell Metab. 2014; 19(3):373-9.

30. Cosin-Roger J, Simmen S, Melhem H, Atrott K, Frey-Wagner I, Hausmann M, de Valliere C, Spalinger MR, Spielmann $\mathrm{P}$, Wenger $\mathrm{RH}$, et al. Hypoxia ameliorates intestinal inflammation through NLRP3/mTOR downregulation and autophagy activation. Nat Commun. 2017:8(1):98.

31. Kearney CJ, Martin SJ. An inflammatory perspective on Necroptosis. Mol Cell. 2017:65(6):965-73.

32. Baxter-Holland M, Dass CR. Doxorubicin, mesenchymal stem cell toxicity and antitumour activity: implications for clinical use. J Pharm Pharmacol. 2018;70(3):320-7.

33. Cagel M, Grotz E, Bernabeu E, Moretton MA, Chiappetta DA. Doxorubicin: nanotechnological overviews from bench to bedside. Drug Discov Today. 2017:22(2):270-81.

34. Imam F, Al-Harbi NO, Al-Harbi MM, Ansari MA, Al-Asmari AF, Ansari MN, AlAnazi WA, Bahashwan S, Almutairi MM, Alshammari M, et al. Apremilast prevent doxorubicin-induced apoptosis and inflammation in heart through inhibition of oxidative stress mediated activation of NF-kappaB signaling pathways. Pharmacol Rep. 2018;70(5):993-1000.

35. Gao Y, Yang H, Fan Y, Li L, Fang J, Yang W. Hydrogen-rich saline attenuates cardiac and hepatic injury in doxorubicin rat model by inhibiting inflammation and apoptosis. Mediators Inflamm. 2016;2016:1320365. 
36. Wang L, Li Y, Deng W, Dong Z, Li X, Liu D, Zhao L, Fu W, Cho K, Niu H, et al. Cardio-protection of ultrafine granular powder for Salvia miltiorrhiza Bunge against myocardial infarction. J Ethnopharmacol. 2018;222:99-106.

37. Chen W, Chen G. Danshen (Salvia miltiorrhiza Bunge): a prospective healing sage for cardiovascular diseases. Curr Pharm Des. 2017;23(34):5125-35.

38. ME XD, Cao YF, Che YY, Li J, Shang ZP, Zhao WJ, Qiao YJ, Zhang JY. Danshen: a phytochemical and pharmacological overview. Chin J Nat Med. 2019;17(1):59-80

39. Chen X, Yu J, Zhong B, Lu J, Lu JJ, Li S, Lu Y. Pharmacological activities of dihydrotanshinone I, a natural product from Salvia miltiorrhiza Bunge. Pharmacol Res. 2019;145:104254.

40. Zhang P, Liu MC, Cheng L, Liang M, Ji HL, Fu J. Blockade of LOX-1 prevents endotoxin-induced acute lung inflammation and injury in mice. J Innate Immun. 2009;1(4):358-65.

41. Liu Q, Zhang Y, Lin Z, Shen H, Chen L, Hu L, Jiang H, Shen X. Danshen extract 15,16-dihydrotanshinone I functions as a potential modulator against metabolic syndrome through multi-target pathways. J Steroid Biochem Mol Biol. 2010;120(4-5):155-63.

42. Lam FF, Yeung JH, Chan KM, Or PM. Dihydrotanshinone, a lipophilic component of Salvia miltiorrhiza (danshen), relaxes rat coronary artery by inhibition of calcium channels. J Ethnopharmacol. 2008; 119(2):318-21.

43. Zhao W, Li C, Gao H, Wu Q, Shi J, Chen X. Dihydrotanshinone I attenuates atherosclerosis in ApoE-deficient mice: role of NOX4/NF-kappaB mediated Lectin-like oxidized LDL Receptor-1 (LOX-1) of the endothelium. Front Pharmacol. 2016;7:418.

44. Guo Y, Wu X, Wu Q, Lu Y, Shi J, Chen X. Dihydrotanshinone I, a natural product, ameliorates DSS-induced experimental ulcerative colitis in mice. Toxicol Appl Pharmacol. 2018:344:35-45.

45. Zhang Y, Li C, Li S, Lu Y, Du S, Zeng X, Chen X, Chen J. Dihydrotanshinone I alleviates crystalline silica-induced pulmonary inflammation by regulation of the Th immune response and inhibition of STAT1/STAT3. Mediators Inflamm. 2019;2019:3427053.

46. Hulsmans M, Clauss S, Xiao L, Aguirre AD, King KR, Hanley A, Hucker WJ, Wulfers EM, Seemann G, Courties G, et al. Macrophages Facilitate Electrical Conduction in the Heart. Cell. 2017;169(3):510-522.e520.

47. Gangadharan C, Thoh M, Manna SK. Inhibition of constitutive activity of nuclear transcription factor kappaB sensitizes doxorubicin-resistant cells to apoptosis. J Cell Biochem. 2009;107(2):203-13.

48. El-Agamy DS, El-Harbi KM, Khoshhal S, Ahmed N, Elkablawy MA, Shaaban AA, Abo-Haded HM. Pristimerin protects against doxorubicin-induced cardiotoxicity and fibrosis through modulation of Nrf2 and MAPK/NF-kB signaling pathways. Cancer Manag Res. 2019;11:47-61.

49. Zhang J, Cui L, Han X, Zhang Y, Zhang X, Chu X, Zhang F, Zhang Y, Chu L. Protective effects of tannic acid on acute doxorubicin-induced cardiotoxicity: involvement of suppression in oxidative stress, inflammation, and apoptosis. Biomed Pharmacother. 2017;93:1253-60.

50. Akolkar G, Bagchi AK, Ayyappan P, Jassal DS, Singal PK. Doxorubicin-induced nitrosative stress is mitigated by vitamin $C$ via the modulation of nitric oxide synthases. Am J Physiol Cell Physiol. 2017;312(4):C418-c427.

51. Sahan-Firat S, Temiz-Resitoglu M, Guden DS, Kucukkavruk SP, Tunctan B, Sari AN, Kocak Z, Malik KU. Protection by mTOR inhibition on Zymosan-induced systemic inflammatory response and oxidative/Nitrosative stress: contribution of mTOR/MEK1/ERK1/2/IKKbeta/IkappaB-alpha/NF-kappaB Signalling pathway. Inflammation. 2018:41(1):276-98.

52. Liu Y, Zhang DT, Liu XG. mTOR signaling in T cell immunity and autoimmunity. Int Rev Immunol. 2015;34(1):50-66.

53. Song $T$, Yao $Y$, Wang $T$, Huang $H, X i a ~ H$. Tanshinone $\| A$ ameliorates apoptosis of myocardiocytes by up-regulation of miR-133 and suppression of Caspase-9. Eur J Pharmacol. 2017;815:343-50.

54. Guo R, Li G. Tanshinone modulates the expression of BCl-2 and Bax in cardiomyocytes and has a protective effect in a rat model of myocardial ischemia-reperfusion. Hellenic J Cardiol. 2018;59(6):323-8.

55. Chen YF, Day CH, Lee NH, Chen YF, Yang JJ, Lin CH, Chen RJ, Rajendran P, Viswanadha VP, Huang CY. Tanshinone IIA inhibits beta-catenin nuclear translocation and IGF-2R activation via estrogen receptors to suppress angiotensin II-induced H9c2 Cardiomyoblast cell apoptosis. Int J Med Sci. 2017;14(12):1284-91.

56. Weichhart T. mTOR as regulator of lifespan, aging, and cellular senescence: a mini-review. Gerontology. 2018;64(2):127-34

\section{Publisher's Note}

Springer Nature remains neutral with regard to jurisdictional claims in published maps and institutional affiliations.

\section{Ready to submit your research? Choose BMC and benefit from:}

- fast, convenient online submission

- thorough peer review by experienced researchers in your field

- rapid publication on acceptance

- support for research data, including large and complex data types

- gold Open Access which fosters wider collaboration and increased citations

- maximum visibility for your research: over $100 \mathrm{M}$ website views per year

At BMC, research is always in progress.

Learn more biomedcentral.com/submissions 